\title{
Currency Cross Rate and Triangular Arbitrage in Nigerian Exchange Market
}

\author{
Bright O. Osu
}

\begin{abstract}
Economic and financial phenomena are analyzed by models based on statistical physics; for example modeling of financial markets as stochastic processes. Stochastic models of stock price changes have been studied by many authors, only that these models discuss change in one price. This paper studies the existence of interaction between the prices of the markets caused by a financial activity called currency cross rate and currency cycle (or triangular arbitrage transaction) as in the Nigerian exchange markets. A simple economic model where the time evolution is described by an equation capturing the currency cross rate under arbitrage is studied. We show that the mean-field limit of this equation as well as the wealth of the investors (the arbitrageurs) in this market is distributed according to the power-law type.
\end{abstract}

Index Terms - Currency cross rate, Power-law distribution, Nigerian exchange market, Triangular arbitrage.

\section{INTRODUCTION}

Like almost anything else, the value of any currency is determined by supply and demand. The greater the demand in relation to the supply, the greater the value, and vice versa. This is true even when no foreign exchange is involved. For instance, if a country never expands its money supply, then the money that is available becomes more valuable as the economy expands. Thus, the price of individual items decreases, which is deflation.

When the money supply expands faster than the economy, then money becomes less valuable. It takes more money to purchase single items, leading to inflation.

Naturally, in foreign exchange, when currency of a particular country is plentiful, it will have less value against other currencies, and vice versa.

Demand for the currency also affects the exchange rate. If a country pays a significantly higher interest rate than another country, or has significantly more investment opportunities or a more stable government, then that country's currency will have greater value than that of the other country.

Cross rates are the exchange rates of a currency with other currencies, and those currencies with each other. Cross rates are equalized among all currencies through a process called triangular arbitrage. Below is a table of key cross rates of some major currencies.

Manuscript received November 30, 2010.
TABLE 1: CROSS RATE OF SOME MAJOR CURRENCIES.

Key Cross Rates for 5/14/2007.

(SourceMarketWatch.com)

$\begin{array}{crrrc}\text { Currency } & \text { USD } & \text { GBP } & \text { EUR } & \text { JPY } \\ \text { USD } & & 0.50443 & 0.73951 & 120.10500 \\ \text { GBP } & 1.98244 & & 1.46603 & 238.10043 \\ \text { EUR } & 1.35225 & 0.68211 & & \\ \text { JPY } & 0.83260 & 0.41999 & 0.61572 & \end{array}$

From this table, we can calculate what the USD is worth in EUR, thus;

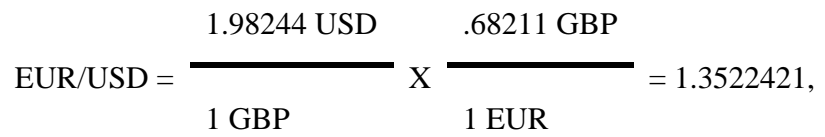

Which is very close to the actual quote, 1.35225 , from the table above. Because we are ignoring the bid/ask spread and transaction costs to simplify the mathematics in this example, there is no reason to believe that it would be exact. It is also true that arbitrage is not a perfect equalizer because the market is not perfectly efficient.

Assuming that exchange rate from 1 NGN-to- USD is 2, one would expect that the rate from USD-to-NGN $=\frac{1}{2}$. Suppose for a second that it was not, but that the rate from USD-to-NGN is 0.6. An investor quickly buys $10 \mathrm{NGN}$ with 5 USD. He could then take his 10 USD and exchange them back for NGN and get back 6 NGN. In this case, he has one more NGN than before. This type of exchange is known as arbitrage.

Since the investor gained a NGN, and since we are not creating or destroying any currency, the rest of the market must have lost a NGN. This of course is bad for the rest of the market. We would expect that the other agents in the currency exchange market will change the exchange rates that they offer so that these opportunities to get exploited are taken away. Still there is a class of investors known as arbitrageurs who try to exploit these differences.

Arbitrage in general takes on more complex forms than this, involving several currencies. Suppose that the NGN-to-USD exchange rate is 2 and the USD-to-GBP is 3. To figure out what the NGN-to-GBP exchange rate needs to be, we just multiply the two rates together:

$$
\begin{aligned}
& \text { NGN - to - GBP }= \\
& (N G N \text { - to - USD) }(\text { USD - to - GBP ). }
\end{aligned}
$$

Equation (1) is an exchange rate's property known as transitivity. To avoid arbitrage we would need the NGN-to-GBP exchange rate to be 6 and the GBP-to-NGN 
exchange rate to be $\frac{1}{6}$. If it were $\frac{1}{5}$, then the investor could again make an additional NGN and the rest of the market would lose one. The arbitrageur in this market transacts whenever he recognizes this opportunities. He makes profit if it takes him a few seconds to recognize the currency circle opportunities and executes the transaction (in this market, the rate from NGN-to-USD is never the same as from USD-to-NGN, etc).

For any three currencies $\mathrm{A}, \mathrm{B}$, and $\mathrm{C}$ trading $\mathrm{A}$ for $\mathrm{B}, \mathrm{B}$ for $\mathrm{C}$ and $\mathrm{C}$ for $\mathrm{A}$ is known as currency circle. A financial activity that takes advantage of three exchange rates among three currencies is known as triangular arbitrage. The triangular arbitrage makes the product of the three exchange rates converge to a certain value (Moosa, 2001 [2]). Aiba, et al, (2002 [1]) had shown that there is in fact triangular arbitrage opportunities in foreign exchange markets and that they generate an interaction among foreign exchange rates.

In the Nigeria exchange market, an agent in the currency market cannot exchange a JPY- to- USD, USD -to- GBP and GBP-to-JPY without making a stop to the NGN, as shown in figure 1 below. This special case of financial activity in the Nigeria exchange market (with Aba as case study) is studied herein. The rest of the paper is organized as follows; the next section establishes the currency cross rate problem. In section three, an analytical approach is used to model a mean-field limit stochastic differential equation which captures the exchange rate. The wealth of the investor is shown to be a power-law type in section four. Section five concludes.

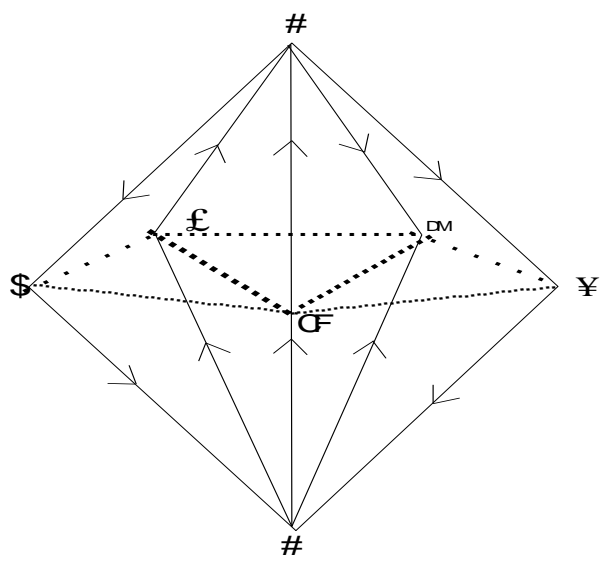

Figure 1: Graphical illustration self-closing triangle of currency exchanges called triangular arbitrage. The doted lines show currency cross rate of more than three currencies. This implies that there exist interactions between more than three currencies in the foreign exchange market.

\section{The CurRency Cross RATE Problem}

There are $n$ types of currencies, and at each exchange one currency is picked at random. Table 2 shows the rate of exchange in NGN in seconds to various currencies, taken from Aba exchange market Abia State, Nigeria, November 2010. How many exchanges one has to make before picking all the currencies? Let $m$ be the number of exchanges made. We bound the probability that $m$ exceeds a certain number and yet not all the currencies are exchanged. Let $C_{i} \in\{1, \ldots, n\}$ be the currency exchanged at $i-t h$ time.
The $j-t h$ time is a success, if $C_{i}$ was not exchanged before at the first $j-1$ time. Figure 3 shows the rate of exchange from NGN to various currencies.

TABLE 2:THE RATE OF EXCHANGE IN SECONDS FROM NGN TO VARIOUS CURRENCIES (SOURCE; ABA FOREIGN EXCHANGE MARKET).

\begin{tabular}{|l|l|l|l|l|l|}
\hline \multicolumn{5}{|c}{ CURRENCIES (SOURCE; ABA FOREIGN EXCHANGE MARKET) } \\
\hline $\begin{array}{l}\text { Rarrency } \\
1 \mathrm{sec}\end{array}$ & Rate 2secs. & Rate 3secs. & Rate 4secs & $\begin{array}{l}\text { Rate } \\
5 \text { secs. }\end{array}$ \\
\hline USD & 146 & 145.50 & 145 & 144 & 151 \\
\hline GBP & 250 & 248.5 & 248 & 243.50 & 245 \\
\hline EUR & 203 & 210 & 208.50 & 208 & 205 \\
\hline WFA & 270 & 271.50 & 274 & 271 & 270 \\
\hline CFA & 248 & 249.50 & 251.50 & 253 & 256 \\
\hline
\end{tabular}

The exchange rate over an interval of time period $\tau=t+\Delta t_{s} \mu(t)$ is given by

$$
(1+\mu(t))=\prod_{i=1}^{n}\left(1+r_{i}(t)\right) \text {. }
$$

For a short time periods (up to a month, say), the one-period exchange rates $r_{\mathrm{i}}(t)$ are small, and $\mu(t)$ can be approximated by:

$$
\mu(t) \cong \sum_{i=1}^{n} r_{i}(t),
$$

with

$$
v=\ln \mu(t)=\sum_{i=1}^{n} \ln \gamma_{i}(t),
$$

If one focuses on the logarithmic rate-change ratio. (Figure 2 shows the probability of the rate product). $r_{\mathrm{T}}(t)$ is geometrically distributed with

$$
E\left[r_{1}(t)\right]=n \ln n+O(n)
$$

and

$$
\lim _{n \rightarrow \infty} \frac{\left.\operatorname{Var}_{n}\left[r_{i}[t]\right)\right]}{n^{2}}=\frac{\pi}{6} .
$$

From USD-to-GBP, one has to get to NGN and vice-versa (as in figure 1 above), that is two edges are created at each step. Thus the total number of edge creations (over all steps) is at most 2 n. Let $C_{i j j}$ denote the amount of currencies that agent $j$ spends exchanging the currency of agent $i$ (and vice-versa). For $n$ types of currencies and $m$ exchanged, there is a relationship between $m$ and the probability of having exchanged at least one currency of the $n$ types. The probability of having exchanged all $n$ currency types abruptly changes from nearly zero to almost one in a small interval centered around $n \ln n$ (Motwani and Raghavan, 1995 [4]). This probabilistic quantity provides some insight into the true behavior of those quantities.

Theorem 1: Let $r_{\mathbb{I}}(t)$ be the exchange for each of the $n$ type's currencies. Then, for any constant $k \in \mathbb{R}$ and $m=n \ln n+k n, \lim _{n \rightarrow \infty} \operatorname{Pr}\left[n_{1}(t)>m\right]=1-e^{-e^{-k}}$.

Proof: See proof theorem 3.8 of Motwani and Raghavan, 1995 [4]. 


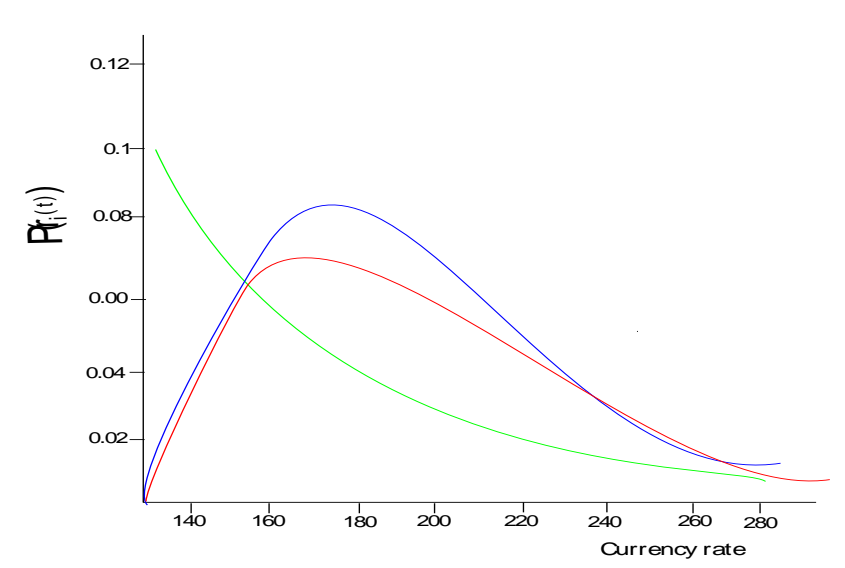

Figure 2 : The probability density function of $r_{i}(t)$. Green for one rate, Blue for two rates and Red for three rates.

\section{ANALYTICAL APPROACH}

The exchange rate of currency $i$ at time $t$ is defined by $r_{\mathbb{I}}(t)$, (time is counted for trading seconds in multiples of a fundamental unit, seconds, say). We define continuous exchange rate as:

$$
\ln \gamma_{1}(t+\Delta t)-\ln \gamma_{\mathrm{i}}(t)=\ln \left(\frac{r_{\mathrm{i}}(t+\Delta t)}{r_{\mathrm{i}}(t)}\right)
$$

and the discrete exchange rate as

$$
\frac{r_{i}(t+\Delta t)-Y_{i}(t)}{r_{i}(t)}=\frac{\Delta r_{i}[t]}{r_{i}(t)} .
$$

As for the log- rates, since we are considering short time period, rates of exchange are small and log-rates are very closely approximated by:

$$
\begin{aligned}
\operatorname{In}\left(\frac{r_{i}(t+\Delta t)}{r_{i}(t)}\right) & =\ln \left(1+\left(\frac{r_{i}(t+\Delta t)}{r_{i}(t)}\right)\right) \\
& \cong\left(\frac{r_{i}(t+\Delta t)}{r_{i}(t)}\right) .
\end{aligned}
$$

From time $t$ to $t+\Delta t$ the exchange rate is characterized by fluctuation, so that

$$
\frac{\Delta r_{i}(t)}{r_{i}(t)}=a(t) \Delta t
$$

due to some environmental effects. Let $a(t)=f_{i}(t)$ +noise as in Osu, (2010 [5]), where $f_{i}(t)$ is an independent fluctuation (a Gaussian random variable with mean $m$ and variance $\left.\sigma^{2}\right)$. Then

$$
\frac{\Delta y_{i}(t)}{r_{i}(t)}=\left(f_{i}(t)+\frac{z^{2}}{3 t \Delta t}\right) \Delta t
$$

Suppose $\tilde{3}_{t} \Delta t \rightarrow N(0, \Delta t)$ and $\tilde{3}_{t} \Delta t=g(\mu(t)) \Delta t$ (where

$g(\mu)$ is an attractive interaction between currencies), then as $\Delta t \rightarrow 0$,

$$
\frac{d r_{i}(t)}{d t}=\left(f_{i}(t)+g(\mu(t)) r_{i}(t)\right.
$$

$$
g(u)=\left(\sum_{\substack{i=1 \\ i \neq j}} C_{i j i}-\varepsilon\right),
$$

so that (12) gives

$$
\frac{d y_{i}(t)}{d t}=f_{i}(t) r_{i}(t)+C\left(\overline{r_{i}(t)}-r_{i}(t)\right) .
$$

All agents exchange with all others at the same rate, that is $c_{i j} \equiv C_{N} \forall i \neq j . \mathrm{N}$ here is the total number of agents(arbitrageur), and $C_{N}$, the scaling needed to make the limit $N \rightarrow \infty$ well defined and $r_{\mathbb{1}} \rightarrow \vartheta r_{\mathbb{1}}$ remains invariant under transformation since the unit of currency is arbitrary when all $r_{\mathrm{f}}^{\mathbb{d}} s$ are multiplied by common arbitrary factor (Bouchaud and Mezard, 2000 [2]). $\overline{r_{1}(t)}=N^{-1} \sum_{\mathrm{i}} r_{\mathbb{1}}$ is the average overall exchange rate. All the agents in this market feel the same influence of their environment, therefore this linear equation (14) is a 'mean-field' model and integrating this equation and summing over $i \overline{\text { gives: }}$

$$
\bar{r}(t)=\bar{r}(0) e^{\left(m+\frac{1}{2} \sigma^{2}\right) t}
$$

Which is deterministic in the limit $N \rightarrow \infty$. Equation (14) can be rewritten in terms of the normalized exchange rate $\mathfrak{J}_{i}={ }^{r_{i}} / \bar{r}$ as:

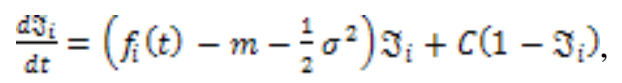

Which we can associate the following Fokker-Planck equation for the evolution of the density of exchange rate $\varphi\left(\mho_{0}, t\right)$ such that:

$$
\frac{\partial \varphi}{\partial t}=\frac{\sigma^{2}}{2} \frac{\partial^{2}}{\partial \mathfrak{J}^{2}} \mathfrak{J}^{2} \varphi+\frac{\partial \varphi}{\partial \mathfrak{Y}}\left[C(\mathfrak{J}-1)+\sigma^{2} / 2 \mathfrak{J}\right] .
$$

It is easy to see that the Pareto distribution $\varphi=\mathrm{FJ}^{-(1+\alpha)} e^{-\left(\frac{\alpha-1}{\mathfrak{J}}\right)}$ solves (17) with

$$
\alpha=1+\frac{c}{\sigma^{2 / 2}},
$$

where $\mathrm{F}=\frac{(\alpha-1)^{\alpha}}{\Gamma(\alpha)}$ is the normalisation factor.

\section{The Distribution OF the WeAlth of the ARBITRAGEUR.}

In this sequel, we drive the distribution for the wealth of the arbitrageur thus;

Theorem 2:

Let $r(t)$ the exchange rate from one currency to another and let $W(t)$ the wealth of an arbitrageur. Then $W(t)$ is said to be distributed according to power-law, if $r(t)$ is stochastically proportional to $W(t)$.

Proof.

Let the relationship between $r(t)$ and $W(t)$ be given as;

$$
r(t)=\theta(t) W(t) \text {, }
$$

where $\theta(t)$ is a stochastic proportionality factor which is uncorrelated with $W(t)$, whose probability density function is given by $\rho(\theta)$, say. We assume here that exchange rate is always positive $(r>0)$. Let

$$
P(r: r>0)=G(r)=\int_{-\infty}^{m} \rho(\theta) H(W) d \theta,
$$


where $G(r)$ and $H(W)$ are the cumulative distributions of $r$ and $W$ respectively. Thus the density function of $r$ is given by

$$
g(r)=\int_{-\infty}^{\infty} \rho(\theta) h\left(\frac{Y}{\theta}\right) \frac{1}{\theta} d \theta, \quad r>0 .
$$

Since the Pareto density function $h(W)$ is non-zero only for $W>W_{0}$, the contribution to the integral in (21) is only for values of $\theta$ such that $\frac{r}{\theta}>W_{0}$. Then $0<\theta<\frac{y}{W_{0}}$. If $\theta<0$ then $\frac{r}{\theta}<0$ and $h\left(\frac{Y}{\theta}\right)=0$; if $\theta>\frac{Y}{W_{0}}$ then $\frac{r}{\theta}<W_{0}$ and again $h\left(\frac{r}{\theta}\right)=0$. Hence, we write equation $(21)$ as;

$$
g(r)=\int_{0}^{\frac{r}{W_{0}}} \rho(\theta) h\left(\frac{r}{\theta}\right) \frac{1}{\theta} d \theta, \quad r>0 .
$$

Employing the Pareto distribution $\varphi=\mathrm{F}^{-(1+\omega)} e^{-\left(\frac{x-1}{y}\right)}$ for $h$, we have:

$$
g(r)=\mathrm{FJ}^{-(1+\alpha)} e^{-\left(\frac{\alpha-1}{\mathfrak{S}}\right)} \int_{0}^{\frac{r}{W_{0}}} \rho(\theta) h\left(\frac{1}{\theta}\right)^{-\alpha} d \theta .
$$

$g(r)$ has important property for being asymptotically Paretian; that is as $r \rightarrow \infty, \quad g(r) \rightarrow F^{-(1+\alpha)} e^{-\left(\frac{\alpha-1}{g}\right)}$, where $F=\mp \int_{0}^{\infty} \rho(\theta) h\left(\frac{1}{\theta}\right)^{-\infty} d \theta$. Thus, the single exchange rate distribution generated by market in which the investor's wealth is distributed according to the Pareto law with exponent $\alpha$ is asymptotically Paretian with the same exponent $\alpha$.

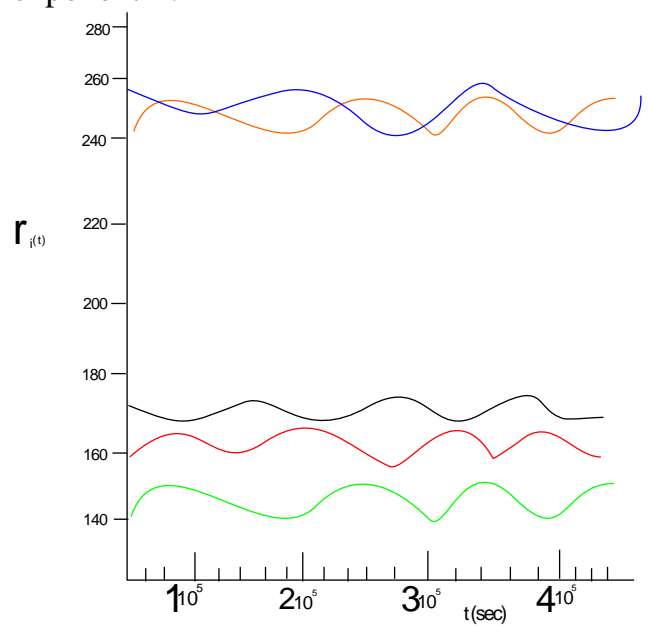

Figure 3: The rate of change in seconds from NGN to various currencies. Green; USD-to-NGN, Red; EUR-to-NGN, Black; CFA-to-NGN, Orange; WFA-to-NGN and Blue; GBP-to-NGN.

\section{CONCLUSION}

We first establish that the currency circle exists in the Aba foreign exchange market with the arbitrageurs making profit whenever there is opportunity and they transact. We further discussed a simple equation capturing the exchange rate between currencies. The mean-field limit of this equation was investigated and shown that the distribution of wealth of arbitrageurs is Paretian.

\section{REFERENCES}

[1] Aiba, Y., Hatano, N., Takayasu, H., Marumo, K., and Shimizu, T., "Triangular arbitrage as an interaction among foreign exchange rates". Physical A. 310: 467-479, 2002.
[2] Bouchaud, J_P., and Mezard, M., “ Wealth condensation in a simple model of economy". arXiv: cond-mat/0002374v1, 2000

[3] Moosa, I., " Triangular arbitrage in the spot and forward foreign exchange markets". Quant. Finance. 1: 387-257, 2001.

[4] Motwani, R. and Raghavan, P. "Randomized algorithms". Cambridge University press, USA, ISBN 0-521-47465-5, pp. 59-63, 1995.

[5] Osu, B. O., "Application of logistic function to the risk assessment of financial asset returns". J. Modern Mathe. Stat. 4(1):7-10, 2010.

Bright O. Osu received a B.Sc. degree in Mathematics from Abia State University of Nigeria in 1998, and then he received an M.Sc.in Mathemtics (probability Theory) from University of Ibadan, Nigeria in 2003 and Ph.D degree in Financial Mathematics from Abia State University of Nigeria, 2008. He works in Department of Mathematics as Lecturer at Abia State University, Uturu, Nigeria.. His research interests include Stochastic Approximation, Mathematics of finance and Probability Theory. 